(2) Open Access Full Text Article

REVIEW

\title{
Ultrasound-guided transversus abdominis plane blocks for analgesia post cesarean section
}

\author{
This article was published in the following Dove Press journal: \\ Comparative Effectiveness Research \\ I3 April 201 I \\ Number of times this article has been viewed
}

\author{
Sara MC Kelly' \\ Rajiv K Malhotra ${ }^{2}$ \\ 'Department of Anesthetics, \\ Russell's Hall Hospital, Dudley, UK; \\ ${ }^{2}$ Department of Anesthetics, Liverpool \\ Women's Hospital, Liverpool, UK
}

Correspondence: Sara MC Kelly Department of Anesthetics, Russell's Hall Hospital, Pensnett Rd, Dudley, West Midlands, DYI2HQ, UK

Tel +44 I 384244076

Email sarakelly7@hotmail.com
Introduction: Cesarean section is a commonly performed operation and postoperative analgesia is needed so the mother can bond and look after her newborn. Transversus abdominis plane (TAP) blocks have been shown to improve postoperative analgesia in laparoscopic cholecystectomy and hysterectomies. The purpose of this article is to review the evidence for the use of TAP blocks in cesarean sections.

Methods: A literature search identified four randomized controlled trials that looked at the use of TAP blocks in cesarean section. Copies were obtained and critically appraised.

Results: Four randomized controlled trials were found that looked at the use of TAP blocks in cesarean sections. Two studies showed that TAP blocks reduced the postoperative analgesic requirements of patients compared to a placebo; one paper showed no difference when TAP blocks were used. In a study comparing TAP blocks with subarachnoid morphine, TAP blocks were associated with poorer postoperative pain scores and higher rescue analgesia. There was no consistent local anesthetic dose used amongst the trials. None of the studies revealed any statistically significant side effects from TAP blocks.

Discussion: TAP blocks have been shown to offer improved pain relief after cesarean sections when compared to a placebo, but have been shown to be inferior to subarachnoid morphine. Standardization of local anesthetic and dosage may result in an improved evidence base from which to draw clinically relevant conclusions.

Keywords: TAP block, cesarean section, regional anesthesia

\section{Introduction}

Cesarean sections account for approximately $24.6 \%$ of all births in the UK. ${ }^{1}$ They are major surgical procedures ${ }^{2}$ that are becoming increasingly common worldwide. Significant discomfort can be expected afterwards: up to $79 \%$ of women experience pain at the incision site that can last for up to 2 months. ${ }^{3}$ Women who have cesarean sections have different postoperative needs from patients undergoing other abdominal surgery. They need to be mobile and observant as soon as possible in order to meet the physical, psychological and emotional needs of their newborn. Anesthesia used for cesarean section includes spinal, epidural and general. Traditionally, analgesia adjuncts used were from either systemic drugs, or by epidural anesthesia. ${ }^{4}$ Blocking the peripheral nerves is an alternative means of providing analgesia that may reduce opioid consumption and therefore dose related side effects.

Transversus abdominis plane (TAP) block is a peripheral nerve block that anesthetizes the abdominal wall. There are three muscle layers of the lateral abdominal wall: the external oblique; the internal oblique; and the transversus abdominis. The central 
abdominal wall also includes the rectus abdominis muscles. The nerve supply to the anterior abdominal wall runs between the internal oblique and the transversus abdominis muscles. ${ }^{5}$ Infiltrating the transversus abdominis plane (TAP) with local anesthetic can block the sensory nerves of the anterior abdominal wall. Direct vision of needle placement is possible with the use of ultrasound which should in theory increase the accuracy and efficacy of these blocks. TAP blocks are now more commonly used in surgery involving the abdominal wall. Studies have shown that they are effective when compared to a placebo in hysterectomy ${ }^{6}$ and laparoscopic cholecystectomy. ${ }^{7}$ The purpose of this article is to review the evidence for their use in cesarean sections.

\section{Methods}

Online databases (PubMed, Medline) were searched for randomized controlled trials that assessed the efficacy of TAP blocks for post-cesarean section analgesia. Search included: TAP/transversus abdominis plane/transversus/abdominis and Cesarean section/Cesarean section/C section/section. Ten studies were found, and six were discarded as they were not randomized controlled trials. The four remaining articles were obtained and critically appraised.

\section{Results}

There are four main papers looking at the use of TAP blocks in cesarean section. They have been reviewed and the main questions from the article are as follows.

\section{Does the use of TAP blocks improve postoperative analgesia requirement?}

There was mixed evidence for this. Two studies ${ }^{8,9}$ found that postoperative analgesia requirements were reduced, whereas the other two studies did not support this need. ${ }^{10,11}$

Belavy et $\mathrm{al}^{8}$ looked at the morphine patient-controlled analgesia (PCA) requirements of patients following a cesarean section. There were 47 participants; all had the same spinal anesthetic and analgesia prescription. Ultrasound-guided TAP blocks with ropivacaine $(0.5 \%)$ were given to 23 women and the remaining 24 had a placebo block with saline. Patients were excluded from the trial if they were regular opioid users or had a BMI $>35$ or weight $<50 \mathrm{~kg}$. Benefits of the TAP block included reduced median total morphine use in 24 hours (18 vs $32 \mathrm{mg}$ ) and increased time to first use of the PCA (median time was three hours compared to two hours in those who had received the saline TAP block). There was no difference in the visual analog scores but patients reported higher satisfaction with their analgesia in the active group.

McDonnell et $\mathrm{al}^{9}$ carried out a double blind randomized controlled trial with a primary intention of looking at analgesia requirements. There were 50 patients in the trial, all of whom had a cesarean section under spinal anesthesia with bupivacaine and fentanyl. They were all given the same analgesia: 25 then had a TAP block with $1.5 \mathrm{mg} / \mathrm{kg}$ ropivacaine $(0.75 \%)$ each side and 25 had a TAP block with normal saline. The landmark technique was used instead of ultrasound-guided technique. Patients included were ASA I-III. (ASA is a classification system adopted by the American Society of Anesthesiologists for assessing preoperative physical status.) Patients were excluded if they were receiving medical therapies considered to result in tolerance to opioids. A standard analgesic regimen was prescribed for every patient including morphine PCA. The presence and severity of pain, nausea, and sedation were assessed at set intervals after TAP blockade. The study showed that the 48-hour total morphine requirements with a PCA were significantly reduced with the intervention. It was also shown that the first morphine requirement was later ( 3 hours 50 minutes) compared to the placebo (90 minutes).

Kanazi et al ${ }^{10}$ did a double blind randomized controlled trial with 57 patients to assess the postoperative experience of women who had subarachnoid morphine (SAM group) compared to those who had a TAP block (TAP group). The TAP was done using bupivacaine $0.375 \%$ plus epinephrine $5 \mathrm{mcg} / \mathrm{mL}$. The study was done on ASA I and II patients, with a BMI less than 35 with no history of chronic pain disorders or alcohol or drug abuse. All of the patients were prescribed standard postoperative analgesia. The time when patients requested breakthrough analgesia was recorded. The main differences in analgesia requirements were noted in the first 12 hours. The median time patients in the TAP group requested analgesia was 4 hours compared to 8 hours in the SAM group. The median number of tramadol doses received between 0 and 12 hours was also lower in the SAM group. There was no statistical difference in the analgesia requirements in the two groups after twelve hours.

Costello et $\mathrm{al}^{11}$ conducted a randomized controlled trial of 100 women, who had a cesarean section under spinal anesthesia. All of the women had an ultrasound-guided TAP block, 50 women with ropivacaine $(0.375 \%)$ and 50 with a placebo. The study looked at postoperative morphine requirements and their visual analog pain score at 6, 12, 24 and 48 hours and again at 6 weeks postpartum. The main conclusion from the trial was that there was no statistically significant difference 
between the two. However on closer inspection of the results during the first 3 hours, more patients who had received the placebo TAP block required morphine earlier.

\section{What is the ideal drug and dose for a TAP block?}

Different drug regimes were used in each study. Both studies that found TAP blocks to be effective used ropivicaine. McDonnell et $\mathrm{al}^{9}$ used a weight-related dose $(1.5 \mathrm{mg} / \mathrm{kg}$ of $0.75 \%$ ropivicaine) and Belavy et $\mathrm{al}^{8}$ used a standard dose ( $20 \mathrm{~mL}$ of $0.5 \%$ ropivacaine each side). Costello et a ${ }^{11}$ didn't find any improvement in the analgesia requirements when he used a lower dose of ropivacaine $(0.375 \% 20 \mathrm{~mL}$ each side $)$. Kanazi et a ${ }^{10}$ used a different drug: their TAP block regime was bupivacaine $0.375 \%$ with ephedrine, which was less effective than subarachnoid morphine in providing analgesia. Studies that used a higher concentration of ropivicaine found that TAP blocks were effective; however none of the studies compared effectiveness of drug doses.

\section{What side effects were experienced by patients in the studies?}

Kanazi et al ${ }^{10}$ compared subarachnoid morphine analgesia compared to TAP block analgesia. Patients in the SAM group had higher levels of nausea than the TAP group ( $46 \%$ compared to $17 \%$ ). Thirty-seven percent of the SAM group also experienced pruritus at 2, 4, 6 and 12 hours, whereas no patients in the TAP group experienced any problems. There was no difference in patient satisfaction or levels of sedation in the two groups.

Costello et al ${ }^{11}$ compared TAP blocks to a placebo. The results showed no difference in side effects shown by patients. This is relevant as TAP blocks may be used as an adjunct analgesia in patients where there is concern about side effects: for example patients who experience vomiting or drowsiness after morphine.

Belavy et $\mathrm{al}^{8}$ and McDonnell et $\mathrm{al}^{9}$ both compared TAP blocks to a placebo. Fewer patients in the active group reported nausea or received antiemetics; this is probably related to their decreased morphine requirements.

\section{At what times were outcomes assessed?}

Kanazi et al $^{10}$ assessed patients earliest; they reviewed the patients at 2, 4, 6 and 12 hours. They found the main differences were in earlier morphine requirements and pain scores before 12 hours postprocedure. As the other studies did not begin to assess pain scores until 6 hours postprocedure, there may be other differences in pain scores not noted. Time to discharge was not assessed by any of the trials.

\section{Discussion}

Evidence from two of the studies suggests that there is a place for the use of TAP blocks following a cesarean section, but there have been no large scale multicentered studies carried out on the efficacy and side effects experienced when used in a cesarean section. Different drugs and doses used in the block resulted in different conclusions being drawn. No studies compared drug doses used.

McDonnell et $\mathrm{al}^{9}$ study using a landmark technique for the TAP showed a greater reduction in morphine use than Belavy et al: a reduction of $70 \%$ in the active group compared to that in the placebo group over 24 hours as opposed to Belavy et al $43 \%$ reduction. ${ }^{8}$ This could be due to a number of reasons: the weight-related dose of the ropivicaine may be more effective, or perhaps as Belavy et $\mathrm{al}^{8}$ hypothesizes, the landmark technique may deliver a more efficient analgesic. This would need further research before conclusions could be drawn. Studies with standardized TAP block dose and methods are needed.

A common theme in the studies was that patients receiving TAP blocks appeared to have less nausea. This is very useful as nausea can prevent the patient from mobilizing and breastfeeding their baby. It may also delay hospital discharge. There may also be a case for using TAP blocks in patients who are more likely to experience postoperative nausea and vomiting. Also patients were excluded from the studies if they regularly used opioids; and this is a particular cohort of people that may find local anesthetic more beneficial than systemic analgesia.

In conclusion our review finds conflicting evidence. There is some evidence that suggests that if TAP blocks are performed with higher concentrations of ropivicaine $(0.5 \%+)$, they are useful in reducing rescue analgesia and associated side effects, but this was not found in all of the studies. There is evidence to suggest that TAP blocks will reduce analgesia requirements post-cesarean section, but large randomized controlled trials are needed in this area to prove that they are effective. It would be interesting to see a large randomized controlled trial of post-cesarean TAP blocks with multiple groups. The groups could all employ different TAP drug regimes, including a placebo. If all of the patients then had analgesia requirements and side effects documented at the same time intervals, it would be possible to assess the efficacy of the differing TAP block doses. For the results to be statistically significant, it would be necessary to see an effect size of at least $15 \%$. Sample size needed would depend on how many drug regimes were 
being compared. The time to discharge from both recovery and hospital could also be compared. Ultrasound-guided TAP blocks are a relatively new method in anesthesia. As techniques and knowledge of the blocks improve they may be more widely used.

\section{Disclosure}

The authors report no conflicts of interest in this work.

\section{References}

1. National Hospital Service. NHS Maternity Statistics, 2008-09. Available from: http://www.ic.nhs.uk. Accessed February 15, 2011.

2. Farragher RA, Laffey JG. Postoperative pain management following cesarean section. In: Shorten G, Carr D, Harmon D, et al, editors. Postoperative Pain Management. An Evidence-based Guide to Practice. 1st ed. Philadelphia, PA: Saunders Elsevier; 2006:225-238.

3. Declercq E, Cunningham DK, Johnson C, Sakala C. Mothers' reports of postpartum pain associated with vaginal and cesarean deliveries: results of a national survey. Birth. 2008;35:16-24.

4. Kehlet H, Dahl JB. The value of "multimodal" or "balanced analgesia" in postoperative pain treatment. Anesth Analg. 1993;77:1048-1056.
5. Netter FH. Back and spinal cord. In: Netter FH, editor. Atlas of Human Anatomy. Summit, NJ. The Ciba-Geigy Corporation; 1989:145-155.

6. Carney J, McDonnell JG, Ochana A, Bhinder R, Laffey JG. The transversus abdominis plane provides effective postoperative analgesia in patients undergoing abdominal hysterectomy. Anesth Analg. 2008;107:2056-2060.

7. El-Dawlatly AA, Turkistani A, Kettner SC, et al. Ultrasound guided transverses abdominis plane block: description of a new technique and comparison with conventional systemic analgesia during laparoscopic cholecystectomy. Br J Anaesth. 2009;102:763-767.

8. Belavy D, Cowlishaw PJ, Howes M, Phillips F, Ultrasound-guided transversus abdominis plane block for analgesia after cesarean delivery. Br J Anaesth. 2009;103(5):726-730.

9. McDonnell JG, Curley G, Carney J, et al. The analgesic efficacy of transversus abdominis plane block after cesarean delivery: randomized controlled trial. Anesth Analg. 2008;106(1):186-191.

10. Kanazi GE, Aouad MT, Abdallah FW, et al. The analgesic efficacy of subarachnoid morphine in comparison with ultrasound guided transversus abdominis plane block after cesarean delivery: a randomized controlled trial. Anesth Analg. 2010;111(2):475-481.

11. Costello JF, Moore AR, Wieczorek PM, Macarthur AJ, Balki M, Carvalho JC. The transversus abdominis plane block, when used as part of a multimodal regimen inclusive of intrathecal morphine, does not improve analgesia after cesarean delivery. Reg Anesth Pain Med. 2009;34(6):586-589.
Comparative Effectiveness Research

\section{Publish your work in this journal}

Comparative Effectiveness Research is an international, peer reviewed open access journal focusing on comparative effectiveness of health care including preventative health care strategies, diagnostic strategies, diagnostic technology, medical devices, drugs, medical technology, health systems and organization. The manuscript management system

\section{Dovepress}

is completely online and includes a very quick and fair peer-review system. Visit http://www.dovepress.com/testimonials.php to read real quotes from published authors. 